\title{
Efeitos da ETCC na função cognitiva de idosos com HIV: uma revisão sistemática
}

\author{
Effects of tDCS on the cognitive function of elderly with \\ HIV: a systematic review
}

\section{Efectos de ETCC en la función cognitiva de los ancianos con VIH: una revisión sistemática}

\author{
Nicole de Almeida Gregório ${ }^{1}$, Renan Gama de Oliveira², Elaine \\ Kakuta ${ }^{3}$, Higor Ranniery Panato Passos ${ }^{4}$, Eduardo Henrique Loreti ${ }^{5}$ \\ 1.Graduando em Fisioterapia, Centro Universitário da Grande Dourados, Dourados-MS, Brasil. \\ https://orcid.org/0000-0003-3276-3320 \\ 2.Graduando em Fisioterapia, Centro Universitário da Grande Dourados, Dourados-MS, Brasil. \\ https://orcid.org/0000-0002-2712-9598 \\ 3.Fisioterapeuta, Mestranda em Ciências da Saúde, Universidade Federal da Grande Dourados, Dourados- \\ MS, Brasil. https://orcid.org/0000-0002-0072-0425 \\ 4.Supervisor de Estágio, Departamento de Fisioterapia, Centro Universitário da Grande Dourados, \\ Dourados-MS, Brasil. https://orcid.org/0000-0002-9808-5895 \\ 5.Professor, Doutorando em Ciências da Saúde - UFGD, Departamento de Fisioterapia, Centro \\ Universitário da Grande Dourados, Dourados-MS, Brasil. https://orcid.org/0000-0002-7821-1145
}

\begin{abstract}
Resumo
Introdução. Nos últimos anos, houve um aumento da prevalência do HIV entre os idosos. A infecção pelo HIV em idosos tem sido acompanhada de comorbidades, como alterações na função cognitiva. Objetivo. Analisar e descrever a resposta terapêutica da ETCC na função cognitiva de idosos com HIV. Método. Os artigos foram pesquisados nas bases de dados Scientific Electronic Library Online (Scielo), PubMed, Web of Science e Physiotherapy Evidence Database (PEDro). Ensaios clínicos randomizados publicados entre 2000 e 2020 e com pontuação maior ou igual a 5 na escala PEDro foram incluídos. A revisão foi registrada no PROSPERO (CRD42021260976). Resultados. A busca resultou em 147 artigos, dos quais 4 foram incluídos. Três estudos usaram ETCC anódica no córtex frontal direito inferior e 1 no córtex pré-frontal dorsolateral esquerdo. Melhoria na função cognitiva foi observada após o uso de tDCS. Conclusões. O uso de ETCC em idosos com HIV mostrou ter um resultado promissor na melhoria da função cognitiva.
\end{abstract}

Unitermos. Estimulação Transcraniana por Corrente Contínua; Envelhecido; Complexo AIDS Demência; Sobreviventes de longo prazo ao HIV; Soropositividade para HIV

\begin{abstract}
Introduction. In recent years, there has been an increase in HIV prevalence among the elderly. HIV infection in the elderly has been accompanied by comorbidities, such as changes in cognitive function. Objectives. To analyze and describe the therapeutic response of tDCS on the cognitive function of elderly people with HIV. Method. The articles were searched in the Scientific Electronic Library Online (Scielo), PubMed, Web of Science, and Physiotherapy Evidence Database (PEDro) databases. Randomized clinical trials published between 2000 and 2020 and with scores greater than or equal to 5 on the PEDro scale were included. The review was registered in PROSPERO (CRD42021260976). Results. The search resulted in 147 articles, of which 4 were included. Three studies used anodic tDCS in the inferior right frontal cortex and 1 in the left dorsolateral prefrontal cortex. Improvement in cognitive function was seen after using tDCS. Conclusions. The use of tDCS in elderly people with HIV has been shown to have a promising result in improving cognitive function.
\end{abstract}

Keywords. Transcranial direct current stimulation; Aging; AIDS Dementia Complex; Longterm HIV survivors; HIV Seropositivity 


\section{RESUMEN}

Introducción. En los últimos años, ha habido un aumento en la prevalencia del VIH entre los ancianos. La infección por VIH en los ancianos se ha acompañado de comorbilidades, como cambios en la función cognitiva. Objetivo. Analizar y describir la respuesta terapéutica de la tDCS sobre la función cognitiva de las personas mayores con VIH. Método. Se realizaron búsquedas en los artículos en las bases de datos Scientific Electronic Library Online (Scielo), PubMed, Web of Science y Physiotherapy Evidence Database (PEDro). Se incluyeron ensayos clínicos aleatorizados publicados entre 2000 y 2020 y con puntuaciones mayores o iguales a 5 en la escala PEDro. La revisión fue registrada en PROSPERO (CRD42021260976). Resultados. La búsqueda resultó en 147 artículos, de los cuales se incluyeron 4. Tres estudios utilizaron tDCS anódico en la corteza frontal inferior derecha y 1 en la corteza prefrontal dorsolateral izquierda. Se observó una mejora en la función cognitiva después de usar tDCS. Conclusiones. Se ha demostrado que el uso de tDCS en personas mayores con VIH tiene un resultado prometedor para mejorar la función cognitiva.

Palabras clave: Stimulación Transcraneal de Corriente Directa; Envejecimiento; Complejo SIDA Demencia; Sobrevivientes de VIH a Largo Plazo; Seropositividad para VIH

Trabalho realizado no Centro Universitário da Grande Dourados, Dourados-MS, Brasil.

\section{INTRODUÇÃO}

Nos últimos anos, houve um aumento da prevalência do vírus da imunodeficiência humana (HIV) entre os idosos. Cerca de $70 \%$ das pessoas que vivem com HIV / AIDS nos EUA têm 50 anos ou mais ${ }^{1}$. Segundo dados do Ministério da Saúde, no Brasil, em 2019, foram notificados 2.260 novos casos de HIV em pessoas com mais de 50 anos $^{2}$.

O aumento da prevalência do HIV na população idosa tem sido acompanhado por comorbidades, como alterações na função cognitiva. Distúrbios neurocognitivos são vistos em até metade dos adultos infectados pelo HIV e com idosos com HIV tem risco três vezes maior de desenvolver alterações neurocognitivas ${ }^{3}$.

O transtorno neurocognitivo associado ao HIV (HAND) está relacionado ao efeito do HIV no sistema nervoso central (SNC). Desencadeando disfunção neuronal, levando ao longo 
prazo à atrofia do cérebro. Existe uma predileção do HIV por estruturas subcorticais, especialmente os gânglios da base e a substância branca profunda, resultando em déficits cognitivos nessas áreas ${ }^{4}$.

Idosos com HAND tendem a ter alterações cognitivas relacionadas a atenção, memória de trabalho, aprendizagem, memória, funções executivas e velocidade de processamento de informações prejudicadas ${ }^{5}$. Assim, é necessárias medidas terapêuticas que possam melhorar a função cognitiva de idosos com HIV, e a Estimulação Transcraniana por Corrente Contínua (ETCC) representa uma abordagem promissora nesse sentido ${ }^{1}$.

A ETCC é uma técnica de neuromodulação segura, bem tolerada e barata que usa corrente elétrica contínua de baixa potência para estimular o cérebro, alterando sutilmente 0 potencial de membrana dos neurônios, tornando-os mais ou menos propensos a disparar, dependendo dos parâmetros de estimulação ${ }^{6}$. Estudos têm demonstrado efeitos benéficos da ETCC na neurocognição7,8. Dessa forma, a ETCC pode ser uma ferramenta de tratamento para a cognição em idosos com HIV.

Relatórios recentes do National Institutes of Health (NIH) enfatizaram a importância dos estudos envolvendo a população idosa com HIV ${ }^{9}$. Com o aumento da longevidade dos indivíduos com HIV e o efeito combinado do envelhecimento e do HIV sobre o funcionamento cognitivo, intervenções são necessárias para reduzir os efeitos dos distúrbios neurocognitivos associados ao HIV. No entanto, 
poucos estudos examinaram os efeitos da ETCC na função cognitiva de idosos com HIV.

Assim, o objetivo desta revisão foi analisar e descrever a resposta terapêutica da ETCC na função cognitiva de idosos com HIV.

\section{MÉTODO}

\section{Bancos de dados e critérios de pesquisa}

Esta revisão sistemática da literatura seguiu o Preferred Reporting Itens for Systematic review and Meta-analysis checklist - PRISMA ${ }^{10}$. A revisão foi registrada no PROSPERO (CRD42021260976) e foi norteado a partir da seguinte questão de pesquisa, por meio da estratégia PICO: pacientes idosos com HIV submetidos à ETCC ativa quando comparados com ETCC sham apresenta melhora da função cognitiva?

Os artigos foram pesquisados nas bases de dados Scientific Electronic Library Online (Scielo), PubMed, Web of Science e Physiotherapy Evidence Database (PEDro). A busca dos artigos ocorreu entre 9 e 11 de outubro de 2020 utilizando os descritores descritos no apêndice I e apenas no idioma inglês. Esses descritores foram estabelecidos primeiro no PubMed e, em seguida, formatadas para cada base de dados individualmente. Além disso, foi pesquisado as referências dos artigos, manualmente, em busca de outros artigos potencialmente relevantes. 
Os artigos obtidos nas bases de dados foram imediatamente exportados para o Rayyan Software ${ }^{11}$ para posterior análise.

\section{Critérios de inclusão e exclusão}

Foram selecionados artigos publicados entre 2000 e 2020, que utilizaram ETCC aplicada em humanos de ambos os sexos, com idade superior a 50 anos e com diagnóstico de HIV; ensaios clínicos randomizados com pontuação igual ou superior a 5 na escala PEDro e estudos usando ETCC isolada ou combinada com outra técnica em comparação com ETCC sham isolada ou associada a outra técnica. Os estudos em modelo animal foram excluídos; estudos que não eram ensaios clínicos; estudos em indivíduos sem HIV; estudos que não foram realizados adultos mais velhos, estudos com resultados pouco claros ou erros.

\section{Seleção dos estudos}

Dois pesquisadores independentes (EK e NG) buscaram os artigos de forma independente e, em seguida, realizaram a análise do título e resumo para identificar os estudos relevantes. Os estudos selecionados foram analisados na íntegra seguindo os critérios de inclusão. Caso houvesse alguma divergência entre os pesquisadores em relação a um artigo, um terceiro pesquisador $(\mathrm{EH})$ seria chamado para analisar o artigo. Além disso, o coeficiente Kapp (k) para concordância entre os avaliadores foi calculado usando o pacote estatístico SPSS versão 17.0. 


\section{Qualidade metodológica e risco de viés}

Para reduzir o risco de viés, os artigos incluídos, foram analisados por dois pesquisadores independentes (HR e NG). Os estudos selecionados na etapa de seleção foram avaliados quanto ao rigor metodológico, a fim de verificar se os métodos e resultados da pesquisa são suficientemente válidos para serem considerados. Nesta etapa foi utilizada a escala PEDro para incluir artigos com boa qualidade metodológica e identificar se os resultados dos artigos são facilmente interpretados ou não, usando uma pontuação de

5 como ponto de corte. A escala PEDro avalia estudos em 11 itens pré-estabelecidos. O primeiro item é um critério adicional e representa a validade externa (ou "potencial de generalização" ou "aplicabilidade" do estudo clínico), não sendo incluído no escore total da escala. Os demais itens analisam dois aspectos da qualidade do artigo: validação interna (itens 2 a 9) e se o artigo contém informações estatísticas suficientes para a interpretação dos resultados (itens 10 e 11). Esses itens são classificados como "aplicáveis" ou "não aplicáveis", gerando uma pontuação total que varia entre 0 e 10 pontos $^{12}$. Se não houvesse consenso, um terceiro pesquisador $(\mathrm{EH})$ atuaria como mediador.

Dois pesquisadores (RG e EK) usaram independentemente a ferramenta da Cochrne Collaboration (Rob 2.0) para avaliar o risco de viés. Se não houvesse consenso, um terceiro revisor $(\mathrm{EH})$ atuaria como mediador. Artigos com alto índice de viés foram excluídos da pesquisa. 


\section{Coleta dos dados}

Os dados, dos artigos, foram coletados em relação à questão inicial (participantes, intervenção, comparação e desfecho) e autores; local de estimulação (ânodo e cátodo); parâmetros ETCC; número de sessões e efeitos adversos. Dois pesquisadores independentes ( $E H$ e $N G$ ) realizaram a busca, para garantir que todos os dados relevantes fossem coletados, para minimizar o risco de erros na transcrição e para garantir a precisão na verificação dos dados coletados. O principal resultado encontrado na coleta de estudos foi a respeito dos efeitos da ETCC na função cognitiva.

\section{RESULTADOS}

No total, foram obtidos 147 estudos, destes, 4 foram selecionados por atenderem aos critérios de inclusão (Figura 1), com um total de 140 participantes, sendo que 72 participantes receberam ETCC ativa e 68 ETCC sham. Os artigos apresentaram risco de viés de moderado para baixo (Figura 2) e apresentavam boa qualidade metodológica pela escala PEDro (Tabela 1 ). Em relação à área estimulada, 3 estudos estimularam o córtex frontal inferior direito e 1 estudo a região do córtex pré-frontal esquerdo dorsolateral (Tabela 1). 
Figura 1. Fluxograma dos artigos analisados.

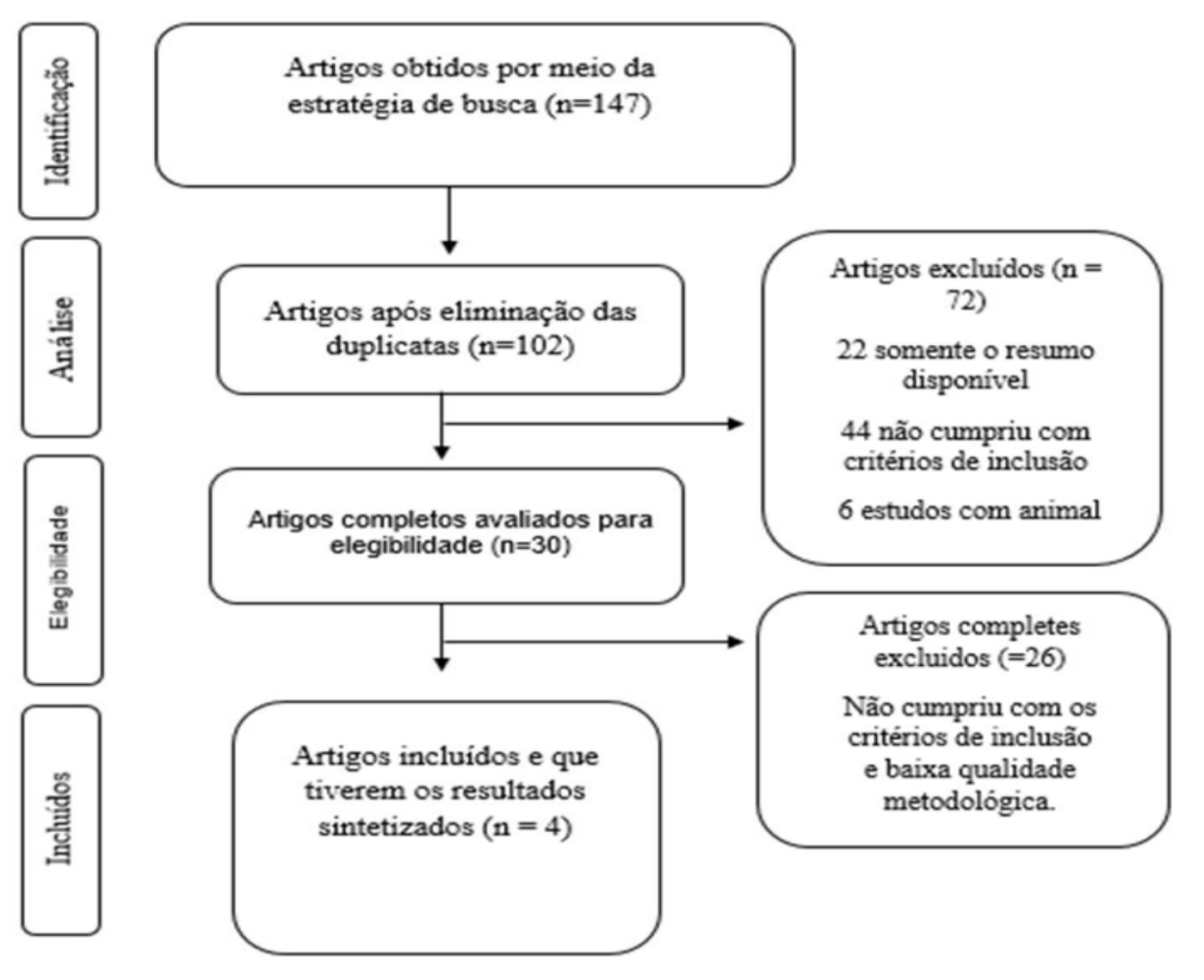

Figura 2. Risco de Viés.

\begin{tabular}{|c|c|c|c|c|c|c|c|c|c|}
\hline Unique ID & $\underline{\text { Study }}$ & Experimental & Comparator & $\underline{\mathrm{D} 1} \underline{\mathrm{D} 2}$ & $\underline{\text { D3 }}$ & $\underline{\text { D4 }}$ & $\underline{\text { D5 }}$ Overall & & \\
\hline 1 & $\overline{\text { Cody et al }}$ & $\mathrm{tDCS}$ & tDCS sham & $\bar{\oplus}$ & $\overline{+}$ & $\overline{1}$ & $\div$ & $\odot$ & Low risk \\
\hline 2 & Pope et al & tDCS+SOP-CRT & tDCS sham+SOP-CRT & $+\div$ & $\odot$ & + & (1) (:) & $:$ & Some concerns \\
\hline 3 & Fazeli et al & tDCS & tDCS sham & $+\oplus$ & + & $\odot$ & $+\odot$ & C & High risk \\
\hline \multirow[t]{6}{*}{4} & Ownby and Acevedo & tDCS & tDCS sham & $4 \div$ & + & 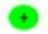 & ( & & \\
\hline & & & & & & & & D1 & Randomisation process \\
\hline & & & & & & & & D2 & Deviations from the intended interventions \\
\hline & & & & & & & & D3 & Missing outcome data \\
\hline & & & & & & & & D4 & Measurement of the outcome \\
\hline & & & & & & & & D5 & Selection of the reported result \\
\hline
\end{tabular}

As percentage (intention-to-treat)

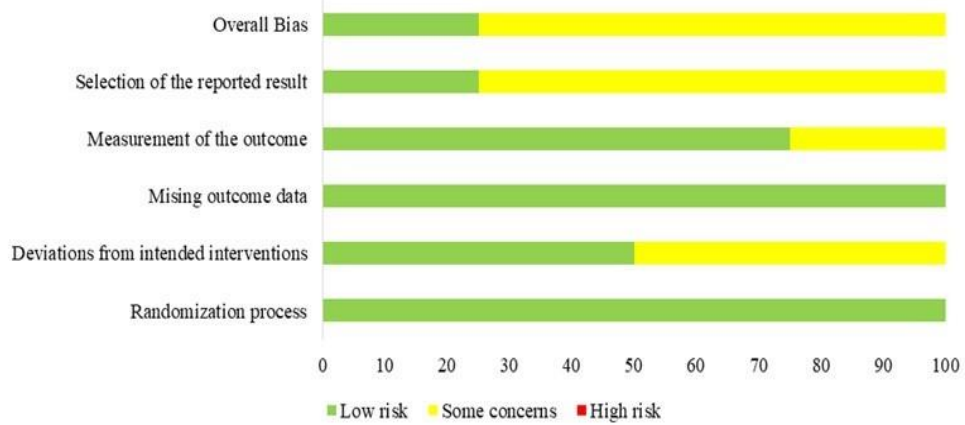


Tabela 1. Características dos artigos analisados.

\begin{tabular}{|c|c|c|c|c|c|c|c|c|c|}
\hline Estudos & $\begin{array}{c}\text { Grupo } \\
\text { ativo (n) }\end{array}$ & $\begin{array}{c}\text { Grupo } \\
\text { simulado } \\
(n)\end{array}$ & $\begin{array}{c}\text { Local de } \\
\text { estimulação } \\
\text { (ânodo) }\end{array}$ & $\begin{array}{c}\text { Local de } \\
\text { estimulação } \\
\text { (cátodo) }\end{array}$ & Parâmetros & $\begin{array}{c}\text { Número } \\
\text { de } \\
\text { sessões }\end{array}$ & Resultados & $\begin{array}{l}\text { Efeitos } \\
\text { adversos }\end{array}$ & PEDro \\
\hline $\begin{array}{c}\text { Cody et al. } \\
(2020)^{13}\end{array}$ & $\begin{array}{c}\text { ETCC HIV } \\
\text { positivo + } \\
\text { SOP (17) / } \\
\text { ETCC HIV } \\
\text { negativo + } \\
\text { SOP (17) }\end{array}$ & $\begin{array}{c}\text { ETCC } \\
\text { simulada } \\
\text { HIV- } \\
\text { positiva } \\
+ \text { SOP } \\
(16) / \\
\text { ETCC } \\
\text { simulada } \\
\text { HIV- } \\
\text { negativa } \\
\text { + SOP } \\
(16) \\
\end{array}$ & $\begin{array}{l}\text { Córtex frontal } \\
\text { inferior direito }\end{array}$ & $\begin{array}{c}\text { Braço } \\
\text { contralateral }\end{array}$ & $\begin{array}{c}2,0 \mathrm{~mA} \\
20 \text { minutos }\end{array}$ & $\begin{array}{c}10 \\
\text { sessões }\end{array}$ & $\begin{array}{l}\text { Os grupos } \\
\text { ETCC ativo } \\
\text { apresentaram } \\
\text { melhora da } \\
\text { função } \\
\text { cognitiva }\end{array}$ & $\begin{array}{c}\text { Não } \\
\text { relatado }\end{array}$ & 8 \\
\hline $\begin{array}{l}\text { Pope et al. } \\
(2018)^{14}\end{array}$ & $\begin{array}{c}\text { ETCC ativo } \\
\text { SOP-CRT } \\
(15)\end{array}$ & $\begin{array}{c}\text { ETCC } \\
\text { sham } \\
\text { SOP-CRT } \\
(15)\end{array}$ & $\begin{array}{l}\text { Córtex frontal } \\
\text { inferior direito }\end{array}$ & $\begin{array}{c}\text { Ombro } \\
\text { contralateral }\end{array}$ & $\begin{array}{c}2,0 \mathrm{~mA} \\
20 \text { minutos }\end{array}$ & $\begin{array}{c}10 \\
\text { sessões }\end{array}$ & $\begin{array}{c}\text { O grupo ETCC } \\
\text { ativo } \\
\text { apresentou } \\
\text { melhora } \\
\text { cognitiva. } \\
\end{array}$ & $\begin{array}{c}\text { Não } \\
\text { reportado }\end{array}$ & 7 \\
\hline $\begin{array}{c}\text { Fazeli et al. } \\
(2017)^{15}\end{array}$ & $\begin{array}{c}\text { ETCC ativo } \\
(17)\end{array}$ & $\begin{array}{c}\text { ETCC } \\
\text { sham } \\
(16)\end{array}$ & $\begin{array}{l}\text { Córtex frontal } \\
\text { inferior direito }\end{array}$ & $\begin{array}{c}\text { Braço } \\
\text { contralateral }\end{array}$ & $\begin{array}{c}2,0 \mathrm{~mA} \\
20 \text { minutos }\end{array}$ & $\begin{array}{c}10 \\
\text { sessões }\end{array}$ & $\begin{array}{c}\text { O grupo ETCC } \\
\text { ativo } \\
\text { apresentou } \\
\text { melhora da } \\
\text { função } \\
\text { cognitiva } \\
\end{array}$ & $\begin{array}{c}\text { Sem } \\
\text { eventos } \\
\text { adversos }\end{array}$ & 9 \\
\hline $\begin{array}{c}\text { Ownby et al. } \\
(2016)^{16}\end{array}$ & $\begin{array}{c}\text { ETCC ativo } \\
(6)\end{array}$ & $\begin{array}{c}\text { ETCC } \\
\text { sham (5) }\end{array}$ & $\begin{array}{l}\text { Córtex pré- } \\
\text { frontal } \\
\text { dorsolateral } \\
\text { esquerdo }\end{array}$ & $\begin{array}{c}\text { Área } \\
\text { supraorbital } \\
\text { direita }\end{array}$ & $\begin{array}{c}1,5 \mathrm{~mA} \\
20 \text { minutos }\end{array}$ & $\begin{array}{c}6 \\
\text { sessões }\end{array}$ & $\begin{array}{c}\text { Não } \\
\text { observaram } \\
\text { diferenças } \\
\text { entre os } \\
\text { grupos, mas } \\
\text { os resultados } \\
\text { são favoráveis } \\
\text { para ETCC. } \\
\end{array}$ & $\begin{array}{c}\text { Não } \\
\text { ralatado }\end{array}$ & 8 \\
\hline
\end{tabular}

SPO: Velocidade de processamento; SOP-CRT: Velocidade de processamento da terapia de remediação cognitiva.

\section{ETCC anódica sobre córtex frontal inferior direito}

Três estudos ${ }^{13-15}$ com um total de 129 participantes foram incluídos, destes 66 com estimulação ETCC ativa e 63 ETCC sham. No estudo de Cody et al. (2020)13 não houve diferenças nas pontuações nos testes de comparação de padrões, substituição de dígitos e Useful Field of View (UFOV) entre os grupos pós-treinamento. Para o Teste de Cópia de Dígitos, um efeito principal para ETCC foi detectado. O grupo de HIV que recebeu ETCC ativa obteve respostas 
significativas quando comparado ao grupo de HIV com ETCC sham.

Pope et al. (2019) ${ }^{14}$ avaliaram os efeitos da ETCC combinada com a terapia de remediação cognitiva de velocidade de processamento (SOP-CRT) para verificar a viabilidade e o impacto na simulação da direção. Os resultados sugeriram um comportamento de direção mais cauteloso após tDCS SOP-CRT ativo, pois os indivíduos randomizados para esse grupo de treinamento mostraram menos desvios de faixa (número de cruzamentos da linha central e excursões nas bordas da estrada) e uma velocidade média de direção mais lenta.

Fazeli et al. (2017) ${ }^{15}$ observaram pouco ou nenhum efeito para a memória de sequência de imagens, vocabulário de imagens, classificação de listas, subteste 2 e 3 do UFOV, total de UFOV, categoria de risco de UFOV e substituição de símbolos de dígitos.

\section{ETCC anódica sobre córtex pré-frontal dorsolateral} (CPFDL)

O estudo de Ownby et al. (2016), com 11 participantes divididos em 6 no grupo ETCC ativo e 5 no grupo ETCC sham foi incluído ${ }^{15}$. Os autores observaram que o grupo ETCC ativa teve uma melhora relativamente maior no aprendizado de acordo com a pontuação total do HVLT. O teste cognitivo antes e depois do treinamento sugere a presença de um efeito positivo da ETCC no aprendizado, memória e velocidade motora em comparação com o treinamento cognitivo sozinho. 


\section{DISCUSSÃO}

A descoberta da Síndrome da Imunodeficiência Adquirida (AIDS) ocorreu em 1981 e, em 1983, o HIV foi isolado. Por ser de fácil transmissão, a AIDS tornou-se uma pandemia, afetando a saúde pública e ganhando notoriedade nas áreas de geriatria e gerontologia ${ }^{17}$.

Com o avanço do tratamento da AIDS, intimamente relacionado à descoberta e distribuição de medicamentos antirretrovirais (TARV), os pacientes infectados têm uma sobrevida mais longa, fazendo com que muitos dos infectados cheguem a uma idade mais avançada. Além disso, a população idosa tem se mantido sexualmente ativa, mas poucos falam abertamente sobre $o$ assunto e sobre as medidas de prevenção ${ }^{18}$.

O aumento da prevalência do HIV na população idosa tem sido acompanhado por comorbidades, como alterações na função cognitiva. Distúrbios neurocognitivos são vistos em até metade dos adultos infectados com HIV, e idosos com HIV têm três vezes mais probabilidade de desenvolver alterações neurocognitivas ${ }^{3}$.

Existem diferentes modelos que explicam a patogênese das doenças HAND. Um dos modelos de patogênese propõe que $o$ vírus atravesse a barreira hematoencefálica transportada pelas células do sistema imunológico, permaneceria latente na microglia até a contagem de células do sistema imunológico cair, com a consequente falha do sistema imunológico em prevenir a multiplicação viral ocorreria os danos neuronais ${ }^{19,20}$. 
Outro modelo propõe que as citocinas produzidas em estágios avançados da AIDS levam a um aumento do fator estimulador de colônias de macrófagos na medula óssea, levando a uma maior proporção de monócitos na medula óssea, que penetram no SNC e maior expressão de fator de necrose tumoral (TNF-a), interleucina-1 $\beta$ (IL-1 $\beta$ ) e complexo de histocompatibilidade classe II. Esse influxo levaria a danos neuronais ${ }^{20}$.

Outro fator relacionado à HAND é a exposição prolongada à TARV que pode predispor o indivíduo a desenvolver lipodistrofia, dislipidemia, diabetes mellitus e doença arterial coronariana, que são fatores que podem levar à Demência de Alzheimer²1,22.

\section{tDCS na função cognitiva}

Há necessidade de intervenções cognitivas na população idosa com HIV ${ }^{23}$. Com o aumento da longevidade populacional, há interesse em avaliar o processo de envelhecimento e os aspectos cognitivos envolvidos, incluindo em pacientes com HIV, bem como investir em protocolos capazes de melhorar a qualidade de vida dos idosos. Porém, como demonstrado, são poucos os estudos que abordam a ETCC na função cognitiva de idosos com HIV.

Estudo tem demonstrado melhora da função cognitiva com uso de ETCC. O córtex pré-frontal, especialmente o córtex pré-frontal dorsolateral, foi sugerido como um alvo para estimulação em estudos que buscam investigar os efeitos da ETCC tanto nos parâmetros motores quanto nos 
aspectos cognitivos de pacientes com doença de Parkinson ${ }^{24}$. Além de melhorias motoras, alguns autores identificaram efeitos benéficos do uso de ETCC anódica aplicada ao córtex pré-frontal função da memória de trabalho, funções executivas, linguagem e cognição global25,26. Assim, a estimulação de DLPFC em pacientes idosos com HIV pode ser benéfica para melhorar a função cognitiva.

A literatura ainda é escassa com relação aos efeitos da ETCC na função cognitiva em idosos ${ }^{27}$, e ainda mais escassa com relação a idosos com HIV. Nesse sentido, descrever como a ETCC melhora a função cognitiva de idosos com HIV se torna um desafio. Fatores genéticos, como o polimorfismo do Fator Neurotrófico Derivado do Cérebro (BDNF), podem estar relacionados com a melhora da função cognitiva após estímulo ${ }^{28}$. No entanto, mais estudos são necessários.

\section{Limitações}

Os estudos mostraram algumas diferenças entre os grupos, o que pode ter interferido nos resultados. No estudo de Cody et al. $(2020)^{13}$, os adultos com HIV eram significantemente mais jovens do que aqueles sem HIV. No grupo com HIV havia significantemente mais minorias raciais e homens quando comparados ao grupo sem HIV.

Os estudos analisados ${ }^{13-15}$ utilizaram estimulação anódica no córtex frontal inferior direito, porém uma abordagem mais focada estimulando o DLPFC poderia ter gerado melhores resultados, uma vez que o DLPFC é uma 
área do córtex frontal relacionada ao processamento cognitivo de ordem superior, como a função executivo.

Apesar de termos extrapolados os critérios de busca com o uso dos descritores, um pequeno número de artigos foi incluído, dificultando a extrapolação dos resultados. Outra limitação é a idade média dos participantes do estudo, sendo: 51,5 anos $^{16} ; 56$ anos no grupo ETCC ativo e 55,63 no grupo ETCC sham ${ }^{15} ; 55,33$ anos no grupo ETCC ativo e 53,73 no grupo ETCC sham ${ }^{14}$ e 55,82 anos no grupo HIV-positivo e 62,12 no grupo HIV-negativo ${ }^{13}$. Assim, a maioria dos participantes do estudo encontrava-se no início da faixa etária geriátrica descrita pelo $\mathrm{CDC}$ e de acordo com o CDC entre 2008 e 2010, um aumento de $26 \%$ dos casos ocorreu em pessoas com 65 anos ou mais. Assim, pesquisas são necessárias para estudar uma faixa etária mais velha ${ }^{28}$.

\section{CONCLUSÃO}

O uso da ETCC em idosos com HIV, de acordo com os artigos analisados, parece ser eficaz na melhora da função cognitiva. No entanto, os resultados devem ser interpretados com cautela, principalmente para aplicação na prática clínica. Sendo necessário mais estudos para se entender os reais efeitos da ETCC na função cognitiva de idosos com HIV.

\section{REFERÊNCIAS}

1.Fazeli PL, Woods AJ, Pope CN, Vance DE, Ball KK. Effect of transcranial direct current stimulation combined with cognitive training 
on cognitive functioning in older adults with HIV: A pilot study. Appl Neuropsychol Adult 2019;26:36-47.

https://doi.org/10.1080/23279095.2017.1357037

2.Brasil. Ministerio da Saúde. Secretaria de Vigilância em Saúde. HIV/Aids. Boletim Epidemiológico (endereço na Internet). Brasília, dez. 2019. (acessado em 2021). Disponível em: http://www.aids.gov.br/pt$\mathrm{br} / \mathrm{pub} / 2019 /$ boletim-epidemiologico-de-hivaids-2019

3. Heaton RK, Clifford DB, Franklin DR, Woods SP, Ake C, Vaida F. HIVassociated neurocognitive disorders persist in the era of potent antiretroviral therapy. Charter Study. Neurology 2010;75:2087-96. https://doi.org/10.1212/WNL.0b013e318200d727

4.Smail RC, Brew BJ. HIV-associated neurocognitive disorder. Handbook Clin Neurol 2018;152:75-97.

https://doi.org/10.1016/B978-0-444-63849-6.00007-4

5.Brew BJ, Crowe SM, Landay A, Cysique LA, Guillemin G. Neurodegeneration and ageing in the HAART era. J Neuroimm Pharmacol 2009;4:163-74. https://doi.org/10.1007/s11481-0089143-1

6.Bikson M, Grossman P, Thomas C, Zannou AL, Jiang J, Adnan T, et al. Safety of transcranial direct current stimulation: Evidence based update. Brain Stimulat 2016;9:641-61.

https://doi.org/10.1016/j.brs.2016.06.004

7.Ferrucci R, Mameli F, Guidi I, Mrakic-Sposta S, Vergari M, Marceglia $S$, et al. Transcranial direct current stimulation improves recognition memory in Alzheimer disease. Neurology 2008;71:493-8. https://doi.org/10.1212/01.wnl.0000317060.43722.a3

8.Kang EK, Kin DY, Paik NJ. Transcranial direct current stimulation of the left prefrontal brain injury: a pilot study. J Rehabil Med 2012;44:346-50. https://doi.org/10.2340/16501977-0947

9.Centers for Disease Control and Prevention. Persons living with diagnosed HIV infection, by year and selected characteristics, 20142018 (endereço na Internet). United States, 2018. (acessaod em: 2021). Disponível em: cdc.gov/hiv/library/reports/hivsurveillance/vol-31/content/living.html

10.Liberati A, Altman DG, Tetzlaff J, Mulrow C, Gøtzsche PC, Loannidis JPA, et al. The PRISMA Statement for Reporting Systematic Reviews and Meta-Analyses of Studies That Evaluate Health Care Interventions: Explanation and Elaboration. PLoS Med 2009;6:132. https://doi.org/10.1136/bmj.b2700

11. Ouzzani M, Hammady H, Fedorowicz Z, Elmagarmid A. Rayyan - a web and mobile app for systematic reviews. BMJ 2016;5:210. https://doi.org/10.1186/s13643-016-0384-4

12. Maher CG, Sherrington C, Herbert RD, Moseley AM, Elkins M. Reliability of the PEDro scale for rating quality of randomizef controlled trials. Phys Ther 2003;83:713-21.

https://doi.org/10.1093/ptj/83.8.713

13.Cody SL, Fazeli PL, Crowe M, Kempf MC, Moneyham L, Stavrinos D, et al. Effects of speed of processing training and transcranial direct current stimulation on global sleep quality and speed of processing in 
older adults with and without HIV: A pilot study. Appl Neuropsychol Adult 2020;27:267-78.

https://doi.org/10.1080/23279095.2018.1534736

14.Pope CN, Stavrinos D, Vance DE, Woods AJ, Bell TR, Ball KK, et al. A pilot investigation on the effects of combination transcranial direct current stimulation and speed of processing cognitive remediation therapy on simulated driving behavior in older adults with HIV. Transp Res Part F Traffic Psychol Behav 2018;58:1061-1073. https://doi.org/10.1016/j.trf.2018.08.002

15. Fazeli $\mathrm{PL}$, Woods $\mathrm{AJ}$, Pope $\mathrm{CN}$, Vance $\mathrm{DE}$, Ball KK. Effect of transcranial direct current stimulation combined with cognitive training on cognitive functioning in older adults with HIV: a pilot study. Appl Neuropsychol Adult 2017;26:36-47.

https://doi.org/10.1080/23279095.2017.1357037

16. Ownby RL, Acevedo A. A pilot study of cognitive training with and without transcranial direct current stimulation to improve cognition in older persons with HIV-related cognitive impairment. Neuropsychiatr Dis Treat 2016;12:2745-54. https://doi.org/10.2147/NDT.S120282

17.Clifford DB, Fagan AM, Holtzman DM, Morris JC, Teshome M, Shah $A R$, et al. CSF biomarkers of Alzheimer disease in HIV-associated neurologic disease. Neurology 2009;73:982-7.

https://doi.org/10.1212/WNL.0b013e3181c5b445

18. Mahmud IC, Cunha LA, Behar PP, Terra NL. O HIV, os idosos e a sexualidade: Uma reflexão sob o olhar da Bioética. Rev SORBI 2018;6:2-30. http://hdl.handle.net/10923/16101

19. Letendre SL, McCutchan JA, Childers ME, Woods SP, Lazzaretto D, Heaton RK, et al. Enhancing antiretroviral therapy for human immunodeficiency virus cognitive disorders. Ann Neurol 2004;56:41623. https://doi.org/10.1002/ana.20198

20.Gartner S. HIV Infection and Dementia. Science 2000;287:602-4. https://doi.org/10.1126/science.287.5453.602

21.Gannon P, Khan MZ, Kolson DL. Current understanding of HIVassociated neurocognitive disorders pathogenesis. Curr Opin Neurol 2011;24:275-83. https://doi.org/10.1097/WCO.0b013e32834695fb

22.Valcour V, Sithinamsuwan $P$, Letendre S, Ances B. Pathogenesis of HIV in the central nervous system. Curr HIV/AIDS 2011;8:54-61. https://doi.org/10.1007/s11904-010-0070-4

23. Weber E, Blackstone K, Woods SP. Cognitive neurorehabilitation of HIV-associated neurocognitive disorders: a qualitative review and call to action. Neuropsychol Rev 2013;23:81-98.

https://doi.org/10.1007/s11065-013-9225-6

24.Boggio P, Khoury LP, Martins DS, Martins OE, Macedo EC, Fregni F. Temporal cortex direct current stimulation enhances performance on a visual recognition memory task in Alzheimer disease. J Neurol Neurosurg Psychiatr 2008;80:444-7.

https://doi.org/10.1136/jnnp.2007.141853

25.Boggio PS, Ferrucci R, Rigonatti SP, Covre P, Nitsche M, PascualLeone $A$, et al. Effects of transcranial direct current stimulation on 
working memory in patients with Parkinson's disease. J Neurolog Sci 2006;249:31-8. https://doi.org/10.1016/j.jns.2006.05.062

26.Doruk D, Gray Z, Bravo GL, Pascual-Leone A, Fregni, F. Effects of tDCS on executive function in Parkinson's disease. Neurosci Lett 2014;582:27-31. https://doi.org/10.1016/j.neulet.2014.08.043

27.Gomes MA, Akiba HT, Gomes JS, Trevizol AP, Lacerda ALT, Dias AM. Transcranial direct current stimulation (tDCS) in elderly with mild cognitive impairment: A pilot study. Dement Neuropsychol 2019;13:187-95. $\quad$ https://doi.org/10.1590/1980-57642018dn13020007

28.Cheeran B, Talelli P, Mori F, Koch G, Suppa A, Edwards M, et al. A common polymorphism in the brain-derived neurotrophic factor gene (BDNF) modulates human cortical plasticity and the response to rTMS. J Physiol 2008;586:5717-25.

https://doi.org/10.1113/jphysiol.2008.159905 